Relations industrielles

Industrial Relations

\title{
Y-a-t-il une politique européenne d'emploi, de sécurité professionnelle et de dialogue social ? Compte rendu de la Conférence EUROCAP, Nantes, février 2006.
}

\section{Hedva Sarfati}

Volume 61, numéro 4, automne 2006

Pistes de renouveau syndical : défis et enjeux

Paths to Union Renewal: Challenges and Issues

URI : https://id.erudit.org/iderudit/014773ar

DOI : https://doi.org/10.7202/014773ar

Aller au sommaire du numéro

Éditeur(s)

Département des relations industrielles de l'Université Laval

ISSN

0034-379X (imprimé)

1703-8138 (numérique)

Découvrir la revue

Citer ce compte rendu

Sarfati, H. (2006). Compte rendu de [Y-a-t-il une politique européenne d'emploi, de sécurité professionnelle et de dialogue social ? Compte rendu de la

Conférence EUROCAP, Nantes, février 2006.] Relations industrielles / Industrial

Relations, 61(4), 748-751. https://doi.org/10.7202/014773ar

Tous droits réservés (C) Département des relations industrielles de l'Université Laval, 2006
Ce document est protégé par la loi sur le droit d'auteur. L'utilisation des services d'Érudit (y compris la reproduction) est assujettie à sa politique d'utilisation que vous pouvez consulter en ligne.

https://apropos.erudit.org/fr/usagers/politique-dutilisation/ 


\section{Y-a-t-il une politique européenne d'emploi, de sécurité professionnelle et de dialogue social?}

Compte rendu de la Conférence EUROCAP, Nantes, février 2006.

Telle semble être la question fondamentale qui sous-tend un projet de recherche européen EUROCAP (20022005) qui s'est achevé par une conférence, à Nantes, les 9 et 10 février 2006, - près de 200 personnes y participaient (universitaires, représentants de pouvoirs locaux, d'entreprises publiques et de syndicats) - pour en tirer les principales leçons. Ce projet a réuni une équipe pluri-disciplinaire d'une trentaine de chercheurs, économistes, sociologues, juristes du travail, historiens, anthropologues, provenant de sept pays européens : Allemagne, Belgique, France, Grande-Bretagne, Italie, Suède et Suisse. Le projet portait sur les stratégies de promotion de l'emploi, de prévention des aléas économiques et sociaux, ainsi que de développement local, formulées et mises en œuvre par différents acteurs - pouvoirs publics à l'échelon national et local, la Commission européenne, les entreprises, les partenaires sociaux. Le projet comportait un volet théorique sur l'approche des capacités, développée par le prix Nobel Amartya Sen, et une série de recherches empiriques des pratiques nationales, locales et d'entreprises vues sous le prisme de cette approche pour évaluer les politiques actuelles de modernisation et de promotion de l' «employabilité » par rapport aux objectifs visés.

Ainsi, par exemple, comme le rappelle le coordinateur du projet, l'économiste Robert Salais (École normale supérieure de Cachan), la modernisation du modèle social européen, objectif que poursuit la Commission européenne, devrait améliorer « la capacité collective à agir et à offrir de nouvelles chances à tous », comme l'affirme l'agenda social européen. Or, constate-il, le projet politique européen actuel ne stimule ni l'économie, ni l'emploi et, malgré les déclarations d'intentions louables (par exemple, l'agenda de Lisbonne), il semble dans la réalité être dépourvu d'une identité sociale concrète (voir R. Salais et R. Villeneuve, dir., Développer les capacités des hommes et des territoires en Europe, Lyon, Éditions ANACT, 2006).

L'approche par les capacités de Sen met l'accent sur les conditions et les moyens permettant une liberté réelle de choix de vie et de travail aux personnes, notamment en matière d'emploi, de développement de carrière et de sécurité des trajectoires professionnelles. Cette approche, comme l'explique Jean-Michel Bonvin, professeur de sociologie à l'Université de Genève qui a participé au projet, associe trois notions que sont les « ressources", les « capacités » et les « fonctionnements ». Les ressources incluent tous les biens et services (marchands ou non) dont peut disposer une personne pour agir, y compris le droit au travail, à l'éducation, à la négociation collective. Mais la possession de ces ressources ne suffit pas pour garantir à la personne une liberté réelle. Celle-ci suppose des capacités d'utiliser ces ressources dans un contexte social et environnemental donné, pour les transformer en droits réels ( « facteurs de conversion »). Enfin, les résultats de l'action dépendent aussi de la manière dont la personne choisit de se comporter effectivement - notion de « fonctionnements » (voir J.-M. Bonvin, «La centralité de la démocratie dans l'approche par les capacités d'Amartya Sen », L'économie politique, juilletseptembre 2005).

Il est difficile de résumer les interventions qui en séance plénière ou en ateliers donnaient une idée de l'étendue et de la profondeur des analyses consacrées tour à tour à : la confrontation du concept d'employabilité avec celui de capacités; l'émergence, à côté d'un 
espace européen unifié par la réglementation et la monnaie unique, de réseaux très étendus de coordination horizontale entre de multiples entreprises et acteurs collectifs publics et privés au niveau local ; l'examen critique des politiques européennes, notamment la stratégie européenne de l'emploi et son influence sur les politiques nationales d'emploi et de protection sociale; et le rôle de la gouvernance d'entreprise et de sa responsabilité sociale comme garant de la sécurité économique. Le texte ci-après ne donne donc que quelques éclairages ponctuels.

Les intervenants à la conférence faisaient état des résultats des enquêtes sur le terrain sur ces diverses dimensions. Simon Deakin, juriste de l'Université de Cambridge, a indiqué les nouvelles limites imposées à l'action régulatrice de l'État par les changements dans les formes prédominantes d'organisation économique et de structures sociales. La prépondérance accrue des marchés et les mutations technologiques tendent de plus en plus à remettre en question les institutions légales traditionnelles de l'État-providence et de la relation d'emploi. En effet, la vision néolibérale qui prédomine aujourd'hui en Europe considère la législation de protection sociale comme limitant l'autonomie contractuelle alors qu'elle est supposée encadrer la coopération contractuelle. Or, argue Deakin, si on admet qu'il faille limiter la liberté contractuelle lorsqu'elle va à l'encontre de valeurs fondamentales (par exemple le maintien de l'ordre public), et si de telles règles sont nécessaires pour protéger le marché, il faut parfois qu'elle protège la société contre le marché. Il importe donc d'identifier les éléments d'un concept de capacités dans ce domaine qui dépasse les garanties formelles d'accès au marché pour incorporer les conditions minimales pour une participation effective dans une société de plus en plus complexe. Alain Supiot, juriste de l'Université de Nantes, a souligné à quel point des zones entières de normes déterminantes échappaient à tout débat public et combien la création d'une connaissance commune sur les réalités du travail était essentielle aujourd'hui en Europe.

Quant à l' «employabilité », pièce maîtresse des politiques de réforme du marché du travail et de l'État social, notamment dans la stratégie européenne pour l'emploi (SEE), elle met l'accent sur la transition des politiques «passives » de protection sociale des salariés vers des politiques actives de retour et du maintien dans l'emploi, parfois au prix d'une précarisation et de pauvreté accrues. Les recherches se demandent de quels emplois il s'agit, quels moyens sont mis en œuvre, quels acteurs sont impliqués et quels liens existent entre le retour à l'emploi et la sécurisation d'un parcours professionnel. Une enquête faite dans une cinquantaine d'entreprises dans sept pays a tenté de cerner les multiples dimensions de l'employabilité comme outil à la fois de gestion de l'aléa économique répondant aux besoins de flexibilité et d'adaptabilité des entreprises et de la liberté de choix du salarié dans le développement de ses compétences et de l'opportunité réelle de les mettre en œuvre. Les entreprises furent examinées selon leur positionnement sur la scène internationale, les restructurations dont elles ont fait l'objet, leur ancrage territorial et sectoriel, la durabilité des produits, la stabilité et la sécurité des parcours professionnels des salariés, l'équité, la participation, la qualité du travail et de l'emploi et l'équilibre entre vie privée et vie professionnelle. Les enquêtes ont montré l'importance pour le développement des capacités de la conjonction de la sécurité, d'opportunités et de supports collectifs permettant aux salariés de se saisir ces opportunités de manière équitable. Or, Christina Garsten, directrice du Département d'anthropologie sociale de l'Université de Stockholm et Bénédicte Zimmermann, sociologue, 
maître de conférences à l'École des hautes études en sciences sociales de Paris, qui ont coordonné ce volet du projet, ont souligné dans leur comparaison des entreprises allemandes, suédoises et françaises, que ces trois caractéristiques se trouvent rarement réunies. En effet, si la sécurité caractérise les entreprises publiques, ces dernières n'offrent que peu d'opportunités, les entreprises privées en difficulté n'offrent ni sécurité, ni opportunités, alors que les entreprises en croissance offrent des opportunités mais rarement des supports collectifs. Dans leur enquête, seules les entreprises qui adhèrent à des valeurs collectives autres que l'efficience conjuguent ces trois exigences, au sein desquelles la capacité des salariés à s'exprimer, participer et accéder à l'information sont primordiales. «L'étendue des capacités varie en fonction du degré d'intégration entre politique du marché du travail, politique sociale et développement économique ».

Remontant du niveau de l'entreprise, le groupe de travail «territoires et capacités » a enquêté sur les systèmes de production locaux, les modes de gouvernance territoriaux et sectoriels et le rôle des services publics locaux dans diverses métropoles européennes (Turin, Le Havre, Marseille) et des zones régionales (West Midlands, Franconie centrale, Brescia, Cracovie, Wroclaw, Leipzig, Nuremberg). Ici l'approche par les capacités s'intéresse aux expériences de démocratie délibérative et à l'émergence de modes coopératifs de gouvernance, parmi lesquels les biens publics produits et offerts localement constituent des ressources à convertir en capacités et servant la collectivité (éducation, transferts technologiques, accès aux marchés mondiaux, etc.). Il s'agissait de voir comment les régions, confrontées à la mondialisation (et à l'élargissement de l'Union européenne) s'adaptent et acquièrent les capacités institutionnelles pour stimuler le développement régional. Parmi les études menées, on signalera la comparaison entre régions britanniques et italiennes dans cinq domaines d'action collective : les relations professionnelles ; les politiques sociales ; l'éducation, la formation et la qualification; les transferts de R$\mathrm{D}$; et les nouveaux produits et marchés. L'enjeu était de démontrer les résultats de deux voies différentes - l'une fondée sur le bas coût du travail (« low road», ou voie secondaire), l'autre s'appuyant sur l'innovation, la connaissance et l'investissement dans les capacités humaines (« high road» ou autoroute). Comme le rappelle le professeur Angelo Pichierri, de la Faculté de sociologie des organisations de l'Université de Turin, « la richesse ou la pauvreté des nations est fonction de la prospérité ou du déclin des systèmes territoriaux subnationaux ». La performance économique des systèmes de production locaux passe par la coopération, la concertation et la délibération institutionnalisées. Mais, constate Pichierri, le développement local optimal peut produire des « externalités » négatives qui menacent sa pérennité. Il rappelle que les améliorations des capacités des entreprises peuvent se faire aussi au dépens des capacités des individus et des régions (c'est la «voie secondaire»). Pour que l'État, les entreprises et les salariés puissent tous améliorer leurs capacités il faut des politiques conscientes, des structures à même d'assurer la mise en œuvre effective, et d'attitudes positives des acteurs.

Remontant au niveau européen, le projet a examiné à la loupe la stratégie européenne de l'emploi (SEE), qui impose des solutions normatives au nom de la rationalité économique, mais, comme l'observe Noël Whiteside, historienne sociale de l'Université de Warwick, elles ne génèrent pas la confiance collective, elles ont une vision très instrumentale de l'employabilité (n'importe quel emploi, durée de contrat ou heures de travail) et fixe des objectifs quantitatifs dont les indicateurs 
posent problème. Ainsi, les procédures de classification ne sont pas fondées sur les faits concrets mais privilégient des processus politiques. Les institutions publiques tendent d'adapter les statistiques pour rehausser leurs performances qui ne correspondent pas à la réalité (agences publiques de l'emploi, hôpitaux, écoles, etc.) En fait, se demande Whiteside, quels sont les véritables objectifs de la SEE : la réduction de la dépendance de l'État-providence ? Une meilleure performance économique ? La création de justice sociale par la garantie d'accès équitable ? Il importe dès lors de savoir si la SEE contribue à améliorer la situation des demandeurs d'emploi en termes de chances d'insertion professionnelle et sociale, et si elle favorise la liberté d'expression effective de chacune des parties prenantes. Et, Whiteside de conclure que le rôle de l'État n'est pas celui d'un PDG, il doit assurer la justice sociale et promouvoir l'égalité des chances des citoyens.

Hedva Sarfati Consultante AISS, Genève

\section{Pension Law, \\ by Ari N. KAPLAN, Toronto: Irwin Law, 2006, 657 pp., ISBN: 1-55221-088-X and ISBN-13: 978-155221-088-8.}

Pension Law is a highly valuable work which constitutes a sourcebook for those (scholars and practitioners alike) who have to deal with such a complicated matter, pension plan governance being structured by labour relations, public policy, and corporate strategy. Moreover, the federal structure of Canadian pension regulation needed a detailed analysis of the foundation and governance of registered pension plans. The most important achievement of the book is to provide a clear, concise, and rigorous understanding of the "basic concepts of the law and regulation of registered pension plans arising from employment in Canada" (p. xxix). Registered pension plans (mostly defined benefit type of plans) are nowadays subject in Canada, like in many western countries, to scrutiny and critics. Even though 5.6 million Canadian workers participated in 14,777 registered employment-based pension plans between 1990 and 2004, pension coverage declined from 45 to 41 per cent, defined benefit plans losing almost 1 million members. Created in the Keynesian and conglomerate business model era, they are now accused of favouring labour market rigidity and nurturing conflicts during the collective bargaining process. The inner problem lies in the institution of Trust as a way to secure retirement income for people involved in asymmetric contractual employment relationships and Welfare State retrenchment.

The book is structured as follows. The first chapter analyses the legal nature of the pension and the second one describes its development. Many sources of pension law exist: contract law, equity and trusts, constitutional law, the Canadian charter of rights and freedoms, administrative law, other legislation like the Income Tax Act, and finally policies and guidelines. As usual, historical foundations are particularly useful in understanding contemporary problems that are questioning pension regulation. The decision in 1965 of the Canadian government to create a public welfare pension scheme implied that provincial legislation had only to set minimum standards for voluntary employer pension plans. However private contractual pension has now become the most important source of retirement income for workers, thus creating a burden on pension law.

The third chapter deals with the scope of pension rights and the fourth one with regulation. Sponsorship, application, 